1 Division of Infectious Diseases and Geographic Medicine, School of Medicine, Stanford University, Palo Alto, CA, USA

2 Division of Infectious Diseases, University of Pennsylvania, Philadelphia, PA, USA

3 Division of Infection and Global Health, School of Medicine, University of St Andrews, St Andrews, UK

Correspondence to: M Cevik mc349@standrews.ac.uk Cite this as: BMJ 2021;374:n2320 http://dx.doi.org/10.1136/bmj.n2320 Published: 23 September 2021

\section{Covid-19 vaccination: evidence of waning immunity is overstated}

\section{The case for universal boosters is weak, and the benefits are unclear}

\author{
Jake Scott, ${ }^{1}$ Aaron Richterman, ${ }^{2}$ Muge Cevik ${ }^{3}$
}

The resurgence of covid-19 in high income countries with advanced vaccine programmes has raised concerns about the durability of vaccine effectiveness, especially against the more transmissible delta variant. This has led some to argue in favour of booster doses for the general population before clear evidence of benefit, which we believe is misguided.

Since the initial randomised trials showed high efficacy of vaccines against a primary endpoint of symptomatic covid-19, ${ }^{1-4}$ observational studies continue to evaluate and report real world performance of vaccines in different contexts and over time..$^{6}$ In addition to providing direct protection against covid-19 disease, available vaccines also substantially reduce transmission, partly by protecting against both symptomatic and asymptomatic infection. ${ }^{7}$ Despite concerns about the immune escape potential of the delta variant, studies consistently indicate that vaccines provide high levels of protection against symptomatic and severe disease as well as death caused by this variant. ${ }^{5}$

With this context in mind, studies with systematic sampling do seem to suggest a modest waning in protection against infection over time. ${ }^{56}$ However, the primary objective of covid-19 vaccines is to protect against severe illness rather than infection, and multiple well designed studies have found sustained vaccine effectiveness against severe covid-19 for most adults. One large UK study, published as a preprint, using a case-control design based on PCR results showed that very high levels of protection against severe disease continued beyond five months after vaccination, especially among people who have no serious underlying conditions.

On the other hand, estimated reductions in vaccine protection against infection vary widely and are more difficult to interpret. These dynamic estimates across studies and countries are heavily influenced by prevalence, behaviour, and circulating variants, and comparing them is not reliable for determining changes in immune protection over time. For instance, while one study from Israel found that the relative rate of infection increased over time after vaccination, several potential biases arise because the timing of vaccination was not random and factors such as risk of exposure to covid-19 and tendency to seek testing confound any association between time since vaccination and infection. ${ }^{9}$ Similarly, while infections among immunised healthcare workers in San Diego, California, increased from June to July, these changes could be explained by increased community prevalence rather than an abrupt waning of immunity. ${ }^{10}$ The effect of behaviour change on measured vaccine effectiveness is also suggested in another US study that found a drop in effectiveness over time only among people under $65 .{ }^{11}$ These examples show the fundamental challenges of assessing effectiveness against infection using routine surveillance data and highlight the need for systematic sampling, consideration of a comprehensive range of measured and unmeasured confounders, and careful interpretation. ${ }^{5}$

\section{Long term immune response}

From an immunological standpoint, plasma neutralising antibody titres are expected to decay eventually following vaccination, but robust and long lived plasmablast and germinal B cell responses have been shown after mRNA vaccination, and memory $B$ cells have been shown to increase over at least six months, improve functionally, and provide cross-variant protection. ${ }^{12} 13$ Plasma neutralising antibody titres may predict some level of protection from symptomatic infection. However, understanding of the strength of this relation over longer periods remains limited. ${ }^{14}{ }^{15}$ Given reported differences in sustained effectiveness against severe disease versus infection, neutralising antibodies are unlikely to be the only mechanism of protection; cellular immunity is more important in long term protection against severe disease. ${ }^{6}$

Most importantly, the long term effect of boosters on admissions remains unknown. Although boosters increase plasma antibody levels and may temporarily extend antibody mediated protection, they have not been shown to augment the memory $\mathrm{B}$ and $\mathrm{T}$ cell responses expected to provide long term protection against severe disease for most immunocompetent people. ${ }^{16}$ In an observational study from Israel reporting benefit associated with a third dose of the Pfizer-BioNTech vaccine (BNT162b2), ${ }^{17}$ the follow-up period in the boosted group was just seven person days for severe disease and 12 person days for infection-too short to assess long term effectiveness. The findings were also highly vulnerable to confounding. ${ }^{17}$ Any potential benefit of additional doses, particularly against symptomatic and severe disease, should be assessed on long term data, ideally from randomised control trials. ${ }^{18}$

Additional vaccine doses are reasonable for people who might not achieve an adequate response to the primary vaccination because of immunosuppression or advanced age, ${ }^{19}$ but overstating evidence of waning immunity for the general population has already had important ramifications, including affecting vaccine confidence. In addition, a focus on waning immunity in high income countries diverts attention and limited vaccine supplies away from the urgent need for primary vaccination of people with reducing infection, transmission, and hospital 
no immunity, particularly in low and middle income countries. This will worsen unacceptable vaccine inequities, prolong the pandemic and its devastating public health and socioeconomic impacts, and increase the risk of new variants. ${ }^{20}$

The large epidemic waves now occurring for the first time during the vaccine era show the ability of more transmissible variants to challenge covid-19 control even in countries with high coverage. This currently poses a greater threat than waning immunity. Demonstration that antibody levels can be boosted in the general population should not be considered evidence of long term effectiveness, and robust clinical data are required to assess the need for additional doses. The risks of remaining unvaccinated are clear and far outweigh the unknown benefits of re-vaccinating the general population. Rapid scale-up of vaccination coverage globally remains the most urgent public health priority.

Competing interests: We have read and understood BMJ policy on declaration of interests and declare MC is a co-opted member of the UK's New and Emerging Respiratory Virus Threats Advisory Group (NERVTAG), which advises the Scientific Advisory Group for Emergencies.

Provenance and peer review: Commissioned; not externally peer reviewed.

1 Polack FP, Thomas SJ, Kitchin N, etalC4591001 Clinical Trial Group. Safety and efficacy of the BNT162b2 mRNA COVID-19 vaccine. N Engl J Med 2020;383:2603-15. doi: 10.1056/NEJMoa2034577 pmid: 33301246

2 Baden LR, El Sahly HM, Essink B, etalCOVE Study Group. Efficacy and safety of the mRNA-1273 SARS-CoV-2 vaccine. N Engl J Med 2021;384:403-16. doi: 10.1056/NEJMoa2035389 pmid: 33378609

3 Voysey M, Clemens SAC, Madhi SA, etalOxford COVID Vaccine Trial Group. Safety and efficacy of the ChAdOx1 nCoV-19 vaccine (AZD1222) against SARS-CoV-2: an interim analysis of four randomised controlled trials in Brazil, South Africa, and the UK. Lancet 2021;397:99-111. doi: 10.1016/S0140-6736(20)32661-1 pmid: 33306989

4 Sadoff J, Gray G, Vandebosch A, etalENSEMBLE Study Group. Safety and efficacy of single-dose Ad26.COV2.S vaccine against covid-19. N Engl J Med 2021;384:2187-201. doi: 10.1056/NEJMoa2101544 pmid: 33882225

5 Tregoning JS, Flight KE, Higham SL, Wang Z, Pierce BF. Progress of the COVID-19 vaccine effort: viruses, vaccines and variants versus efficacy, effectiveness and escape. Nat Rev Immunol 2021:1-11. doi: 10.1038/s41577-021-00592-1. pmid: 34373623

6 Cevik M, Grubaugh ND, Iwasaki A, Openshaw P. COVID-19 vaccines: Keeping pace with SARS-CoV-2 variants. Cell 2021:S0092-8674(21)01057-6. doi: 0.1016/j.cell.2021.09.010. pmid: 34534444

7 Richterman A, Meyerowitz EA, Cevik M. Indirect protection by reducing transmission: ending the pandemic with SARS-CoV-2 vaccination. Open Forum Infect Dis 2021. doi: 10.1093/ofid/ofab259

8 Andrews N, Tessier E, Stowe J, et al. Vaccine effectiveness and duration of protection of Comirnaty, Vaxzevria, and Spikevax against mild and severe COVID-19 in the UK. 2021. [Preprint.] https://khub.net/documents/135939561/338928724/Vaccine+effectiveness+and+duration+of+protection+of+covid+vaccines+against+mild+and+severe+COVID-19+in+the+UK.pdf/10dcd99c0441-0403-dfd8-11ba2c6f5801

9 Goldberg Y, Mandel M, Bar-on YM, etal. Waning immunity of the BNT162b2 vaccine: a nationwide study from Israel.medRxiv 2021. [Preprint.] doi: 10.1101/2021.08.24.21262423.

10 Keehner J, Horton LE, Pfeffer MA, etal. SARS-CoV-2 infection after vaccination in health care workers in California. N EnglJ Med2021;384:1774-5. doi: 10.1056/NEJMc2101927 pmid: 33755376

11 Rosenberg ES, Holtgrave DR, Dorabawila V, etal. New covid-19 cases and hospitalizations among adults, by vaccination status - New York, May 3-July 25, 2021. MMWR Morb Mortal Wkly Rep 2021;70:1150-5. doi: 10.15585/mmwr.mm7034e1 pmid: 34437517

12 Turner JS, O'Halloran JA, Kalaidina E, etal. SARS-CoV-2 mRNA vaccines induce persistent human germinal centre responses. Nature 2021;596:109-13 doi: 10.1038/s41586-021-03738-2 pmid: 34182569

13 Goel RR, Painter MM, Apostolidis SA, etal. mRNA vaccination induces durable immune memory to SARS-CoV-2 with continued evolution to variants of concern.bioRxiv2021. [Preprint.] doi: 10.1101/2021.08.23.457229

14 Khoury DS, Cromer D, Reynaldi A, etal. Neutralizing antibody levels are highly predictive of immune protection from symptomatic SARS-CoV-2 infection. Nat Med 2021;27:1205-11. doi: 10.1038/s41591-021-01377-8 pmid: 34002089

15 Gilbert PB, Montefiore DC, McDermott A, etal. Immune correlates analysis of the mRNA-1273 COVID-19 vaccine efficacy trial.medRxiv 2021. [Preprint.] doi: 10.1101/2021.08.09.21261290

16 Goel RR, Painter MM, Apostolidis SA, etalUPenn COVID Processing Unit. mRNA Vaccination induces durable immune memory to SARS-CoV-2 with continued evolution to variants of concern bioRxiv 2021:2021.08.23.457229. doi: 10.1101/2021.08.23.457229. pmid: 34462751

17 Bar-On YM, Goldberg Y, Mandel M, etal. Protection of BNT162b2 vaccine booster against covid-19 in Israel. N Engl J Med 2021. doi: 10.1056/NEJMoa2114255 pmid: 34525275
18 Thomas SJ, Moreira ED, JrKitchin N, etalC4591001 Clinical Trial Group. Safety and efficacy of the BNT162b2 mRNA covid-19 vaccine through 6 months. N Engl J Med 2021;15. doi: 10.1056/NEJMoa2110345. pmid: 34525277

19 Kamar N, Abravanel F, Marion O, Couat C, Izopet J, Del Bello A. Three doses of an mRNA covid-19 vaccine in solid-organ transplant recipients. N Engl / Med 2021;385:661-2. doi: 10.1056/NEJMc2108861 pmid: 34161700

20 Kiernan S, Galal B, Bollyky TJ. Wanted: one billion booster shots. Think Global Health 2021. https://www.thinkglobalhealth.org/article/wanted-one-billion-booster-shots

This article is made freely available for use in accordance with BMJ's website terms and conditions for the duration of the covid-19 pandemic or until otherwise determined by BMJ. You may use, download and print the article for any lawful, non-commercial purpose (including text and data mining) provided that all copyright notices and trade marks are retained. 